\author{
Ales Krouzecky \\ Jiri Chvojka \\ Roman Sykora \\ Jaroslav Radej \\ Thomas Karvunidis \\ Ivan Novak \\ Jiri Ruzicka \\ Zuzana Petrankova \\ Jiri Benes \\ Lukas Bolek \\ Martin Matejovic
}

\section{Regional cooling of the extracorporeal blood circuit: a novel anticoagulation approach for renal replacement therapy?}

Received: 4 July 2008

Accepted: 25 August 2008

Published online: 18 September 2008

(C) The Author(s) 2008. This article is published with open access at Springerlink.com

J. Chvojka and R. Sykora have equally contributed to this study.

\author{
A. Krouzecky · J. Chvojka · R. Sykora · \\ J. Radej - T. Karvunidis · I. Novak · \\ M. Matejovic ( $)$ \\ ICU, 1st Medical Department, \\ Charles University Medical School \\ and Teaching Hospital, alej Svobody 80, \\ 30460 Plzen, Czech Republic \\ e-mail: matejovic@fnplzen.cz \\ Tel.: +420-37-7103501 \\ Fax: +420-37-7103506 \\ J. Ruzicka $\cdot$ Z. Petrankova $\cdot$ J. Benes · \\ L. Bolek \\ Department of Biophysics, \\ Faculty of Medicine in Plzen, \\ Charles University in Prague, \\ Karlovarska 48, 30166 Plzen, \\ Czech Republic
}

Abstract Objective: To test the hypothesis that cooling of blood in the extracorporeal circuit of continuous veno-venous hemofiltration $(\mathrm{CVVH})$ enables to realize the procedure without the need of anticoagulation. Design: Experimental animal study. Methods: We developed the device for selective cooling of extracorporeal circuit $\left(20^{\circ} \mathrm{C}\right)$ allowing blood rewarming $\left(38^{\circ} \mathrm{C}\right)$ just before returning into the body. Twelve anesthetized and ventilated pigs were randomized to receive either $6 \mathrm{~h}$ of $\mathrm{CVVH}$ with application of this device (COOL; $n=6$ ) or without it (CONTR; $n=6)$. Measurements: Before the procedure and in $15,60,180$, 360 min after starting hemofiltration variables related to: (1) circuit patency [time to clotting (TC), number of alarm-triggered pump stopping (AS), venous and transmembranous circuit pressures (VP, TMP)], (2) coagulation status in the extracorporeal circuit [thrombin-antithrombin complexes $\left(\mathrm{TAT}_{\text {circ }}\right)$, thromboelastography (TEG)] and (3) animal status (hemodynamics, hemolysis and biochemistry) were assessed.
Results: The patency of all circuits treated with selective cooling was well maintained within the observation period. By contrast, five of six sessions were prematurely clotted in the untreated group. As a result, the number of AS was significantly higher in the CONTR group. In-circuit thrombus generation in CONTR group was associated with a markedly increasing $\mathrm{TAT}_{\text {circ }}$. TEG performed at $180 \mathrm{~min}$ of the procedure revealed a tendency to a prolonged initial clotting time and a significant decrease in clotting rate of in-circuit blood in the COOL group. No signs of repeated cooling/rewarming-induced hemolysis were observed in animals treated with "hypothermic circuit" CVVH. Conclusion: In this porcine model, regional extracorporeal blood cooling proved effective in preventing in-circuit clotting without the need to use any other anticoagulant.

Keywords Renal replacement therapy · Anticoagulation . Hypothermia - Hemofiltration . Cooling · Critically ill

\section{Introduction}

A contact of blood with synthetic materials of the extracorporeal circuit results in the activation of coagulation
[1]. Therefore, renal replacement therapy use in the critically ill usually requires administration of anticoagulants. The most common way of preventing blood from clotting is systemic administration of anticoagulants, which, 
however, is not feasible in at risk patients. In these cases, alternative methods are often used including regional anticoagulation, minimal heparinization, use of prostaglandins or circuit saline flushes at regular intervals [2,3]. Unfortunately, none of these methods is optimal, as it is costly, inadequately effective, or associated with metabolic risks [2].

Lowering blood temperature is known to reduce blood clotting $[4,5]$. This effect is mainly due to inhibition of enzymatic reactions of the coagulation cascade and to impaired platelet function $[6,7]$. The lower blood temperature, the more appreciable the effect [5]. On the other hand, blood rewarming seems to normalize the coagulation defect [8]. This has led to a hypothesis under which, if the temperature of the blood in the extracorporeal circuit could be reduced adequately and rewarmed accordingly prior to returning it back to the patient's body, there would be no need for anticoagulation at all.

We therefore conducted an experimental study designed to determine whether cooling of blood in CRRT extracorporeal circuit to $20^{\circ} \mathrm{C}$ with its subsequent rewarming to baseline temperature before returning it to the body will allow performing an extracorporeal procedure without anticoagulant use.

\section{Methods}

The experiment was performed in compliance with the National Institutes of Health Guidelines on the Use of Laboratory Animals. The study protocol was approved by the University Animal Care Committee.

\section{Animals and preparations}

Twelve domestic pigs with a comparable body weight [37 $(33 ; 43) \mathrm{kg}]$ were investigated. Anesthesia was induced with i.v. atropine (0.5 mg), propofol $2 \%(1-2 \mathrm{mg} / \mathrm{kg}$ ) and ketamine $(2 \mathrm{mg} / \mathrm{kg})$. Animals were mechanically ventilated $\left(\mathrm{FiO}_{2} 0.4\right.$, PEEP $5 \mathrm{~cm} \mathrm{H}_{2} \mathrm{O}$, tidal volume $10 \mathrm{ml} / \mathrm{kg}$, respiratory rate adjusted to maintain end-tidal $P \mathrm{CO}_{2}$ between 4.0 and $5.0 \mathrm{kPa}$ ). During instrumentation, surgical anesthesia was maintained with continuous intravenous thiopental $[10 \mathrm{mg} /(\mathrm{kg} \mathrm{h})]$ and repeated boluses of buphrenorphine $(0.3 \mathrm{mg})$. Thereafter, continuous thiopental $[5 \mathrm{mg} /(\mathrm{kg} \mathrm{h})]$ was maintained until the end of the experiment and buphrenorphine $(0.3 \mathrm{mg})$ was added every $3 \mathrm{~h}$ to prevent inadequate analgesia. Muscle paralysis was achieved with pancuronium [0.2 mg/ $(\mathrm{kg} \mathrm{h})$. Infusion of Plasma Lyte solution (Baxter Healthcare) of $10 \mathrm{ml} /(\mathrm{kg} \mathrm{h})$ was administered during the instrumentation and subsequently reduced to $5 \mathrm{ml} /(\mathrm{kg} \mathrm{h})$ as a maintenance fluid.
A 14 Fr double-lumen hemodialysis catheter of $15 \mathrm{~cm}$ length (Arrow International, Inc.; Reading, USA) was inserted via the right femoral vein. The left femoral artery was cannulated by a fiberoptic catheter equipped with a thermistor for blood pressure recording, blood sampling, continuous blood temperature recording, and thermal-dye double-indicator dilution measurements. A cystostomy catheter for urine derivation was placed percutaneously under ultrasound guidance. A post-instrumentation stabilization period of $1 \mathrm{~h}$ was allowed before baseline measurements were obtained.

\section{CRRT setting}

CRRT was performed in a continuous hemofiltration mode (CVVH, MultiFiltrate, Fresenius Medical Care AG \& Co. KGaA, Bad Homburg, Germany) using a polysulfone membrane (Ultraflux AV600S, Fresenius Medical Care AG \& Co. KGaA, Bad Homburg, Germany). Blood flow rate was set at $150 \mathrm{ml} / \mathrm{min}$. A lactate-buffered replacement fluid (Medisol K4, solution for hemofiltration, Medites Pharma, Roznov pod Radhostem, Czech Republic) was administered in a post-dilutional fashion. The treatment was zero-balanced at an ultrafiltration rate $20 \mathrm{ml} /(\mathrm{kg} \mathrm{h})$. To avoid a possible effect of ultrafiltrationmediated changes in measured variables, the substitution and ultrafiltration pumps were temporarily switched off 5 min before every blood sampling.

\section{Cooling device}

Technical schema of the cooling device is shown in Fig. 1. The device was developed in our institution and is currently patent pending (provisional patent number PV 2008-35). Briefly, the device comprises a cooling unit placed under the CRRT system and a second, warming, unit placed behind it. The first heat exchanger is connected to a tube bringing in the cooling medium, while the other is connected to a tube bringing in a heating medium. The temperature in both exchangers can be set across a wide range. In our project, the temperature of the blood at the inflow tract of the extracorporeal circuit was set at $20^{\circ} \mathrm{C}$ while raising it to the baseline body core temperature on the return. The system is fitted with thermal needle sensors, introduced into the circuit via several sampling ports to check whether the preset temperature has been reached. Before each experiment, the sensors were tested using the calibration device Techne TECAL $140 \mathrm{H}$ to check their accuracy. Data from the sensors are continuously transmitted to a computer control unit, automatically adjusting, by a feedback mechanism, the process of cooling and warming both via a change in the cooling/heating medium temperature and change in the rate of medium flow through the heat exchanger. 
Protocol and measured parameters

Twelve pigs were randomized to undergo either $6 \mathrm{~h}$ of "hypothermic circuit" CVVH (COOL; $n=6)$ or "isothermic circuit" CVVH (CONTR; $n=6$ ). Before the beginning of CVVH as well as 15, 60, 180,360 min after its start, variables related to circuit patency (time to clotting, number of alarm-triggered pump stopping, circuit's venous and transmembranous pressures) were recorded. In addition, parameters of coagulation status in the extracorporeal circuit (thrombin-antithrombin complexes measured at 15, 60, 180, 360 min (Enzygnost TAT micro, Behringwerke AG, Marburg, Germany), thromboelastography [measured at 180 min $\left(\right.$ TEG $^{\circledR} 5000$ Thromboelastograph ${ }^{\circledR}$ Hemostasis Analyzer, Haemoscope, USA)] and markers of safety of the method (hemodynamic status, body core temperature, blood count, free plasma hemoglobin, bilirubin and liver enzymes, blood gases) were measured. The TEG measurements were performed at the same temperature as was the temperature in the extracorporeal circuit (i.e. at $20^{\circ} \mathrm{C}$ in "hypothermic" circuit group, at $38^{\circ} \mathrm{C}$ in "normothermic" circuit group).

\section{Statistical analysis}

All values shown are median and interquartile range. Differences within each group during CVVH were tested using a Friedman ANOVA on ranks. Differences between the groups were analyzed using Mann-Whitney analysis.
Fig. 1 Technical schema of the cooling device. $D$ CRRT machine (CVVH mode), V1 cooling unit, $V 2$ warming unit, $P 1$ unit for cooling of the medium, $P 2$ unit for heating of the medium, $C$ pump, $T 1-T 5$ thermal sensors, $R$ computer control unit, $S$ TEG and TAT circ $_{\text {c }}$ sampling point

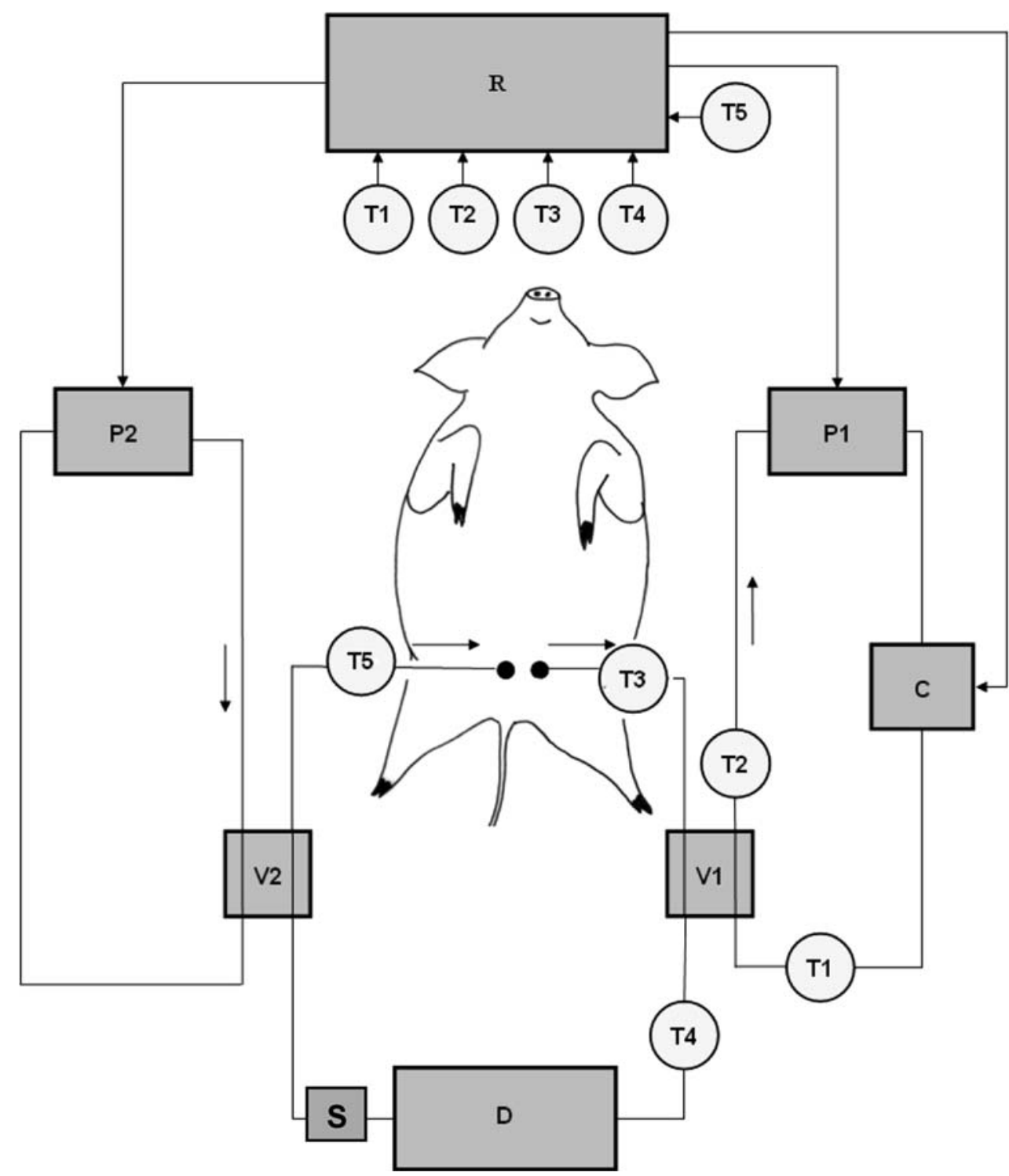


Table 1 In-circuit pressures and thrombin-antithrombin complexes

\begin{tabular}{llllll}
\hline Variable & Group & 15 & 60 & 180 & 360 \\
\hline VP $(\mathrm{mmHg})$ & COOL & $110(88 ; 122)$ & $100(78 ; 158)$ & $80(80 ; 125)$ & $80(80 ; 205)$ \\
& CONTR & $140(110 ; 180)$ & $265^{\mathrm{a}, \mathrm{b}}(200 ; 360)$ & $360^{\mathrm{a}, \mathrm{b}}(315 ; 418)$ & - \\
TMP $(\mathrm{mmHg})$ & COOL & $10(8 ; 10)$ & $10(8 ; 10)$ & $10(10 ; 28)$ & $32(10 ; 48)$ \\
& CONTR & $1(1 ; 20)$ & $5^{\mathrm{b}}(1 ; 10)$ & $30^{\mathrm{b}}(20 ; 285)$ & - \\
TAT circ $(\mu \mathrm{g} / \mathrm{l})$ & COOL & $14(9 ; 23)$ & $44^{\mathrm{b}}(37 ; 49)$ & $70^{\mathrm{b}}(47 ; 141)$ & $104^{\mathrm{b}}(47 ; 110)$ \\
& CONTR & $30^{\mathrm{a}}(21 ; 37)$ & $78^{\mathrm{b}}(57 ; 92)$ & $174^{\mathrm{b}}(121 ; 192)$ & - \\
\hline
\end{tabular}

$V P$ venous pressure, TMP transmembranous pressure, TAT thrombin-antithrombin complexes

${ }^{\text {a }} P<0.05$ versus $\mathrm{COOL}$

b $P<0.05$ versus 15

\section{Results}

Animals in both groups did not differ in any of the measured variables at baseline.

\section{Circuit patency}

The patency of all circuits treated with selective cooling was well maintained within the observation period. By contrast, five of six sessions were prematurely clotted in the untreated group [time to clotting $197(180 ; 240) \mathrm{min}$ ]. As shown in Table 1, progressive circuit obstruction in the control group was accompanied by increasing circuit venous and transmembranous pressures, whereas these variables remained unchanged during the entire session in the COOL group. As a result, the number of pressure alarm-triggered pump stopping was significantly higher in the control group [CONTR $6(5 ; 7)$ vs. COOL $1.5(0 ; 2)$, $P=0.015]$.

Coagulation status in the extracorporeal circuit

The early clotting of the extracorporeal circuit observed in the CONTR group was associated with a progressive

Table 2 In-circuit thromboelastography

\begin{tabular}{lll}
\hline Parameter & Group & \\
\cline { 2 - 3 } & COOL & CONTR \\
\hline$R$ (min) & $19(10.4 ; 19.8)$ & $7.5(1.4 ; 14.1)$ \\
$K(\min )$ & $7.9(6.3 ; 9.4)$ & $3.6^{\mathrm{a}}(1.8 ; 5.5)$ \\
$\alpha$ angle $\left({ }^{\circ}\right)$ & $31(26 ; 34)$ & $52^{\mathrm{a}}(37 ; 67)$ \\
MA (mm) & $77(75 ; 81)$ & $72(54 ; 77)$ \\
\hline
\end{tabular}

$R$ time from initiation of the test to the initial fibrin formation, $K$ time from beginning of clot formation until the amplitude reaches $20 \mathrm{~mm}$ (represents dynamics of clot formation), $\alpha$ angle between the line in the middle of the TEG tracing and the tangential to the "body" of the TEG tracing (represents the kinetics of fibrin build up and cross-linking), $M A$ the rate of amplitude reduction $60 \mathrm{~min}$ after MA (represents the stability of the clot)

${ }^{a} P<0.05$ versus COOL increase in thrombin-antithrombin complexes $\left(\mathrm{TAT}_{\text {circ }}\right)$, suggesting ongoing in-circuit thrombin generation (Table 1). By contrast, the rise in $\mathrm{TAT}_{\text {circ }}$ was less obvious in the COOL group. Concerning the dynamics of in-circuit clot formation, data obtained from thromboelastography (TEG) at $180 \mathrm{~min}$ of the procedure revealed a tendency to a prolonged initial clotting time $(R)$ and a significant decrease in clotting rate $(K, \alpha$ angle) in the COOL group (Table 2), without affecting clot strength (MA). Representative TEG tracings are shown in Fig. 2.

Animal status and biochemistry

During the whole study period all animals in both groups remained hemodynamically stable with unchanged baseline body core temperature. The number of platelets decreased significantly in both groups without any intergroup differences (Table 3). We did not observe any alterations in liver enzymes and blood gases (data not shown). In addition, no signs of repeated cooling/ rewarming-induced hemolysis in serial free plasma hemoglobin measurements were observed in two

(a)

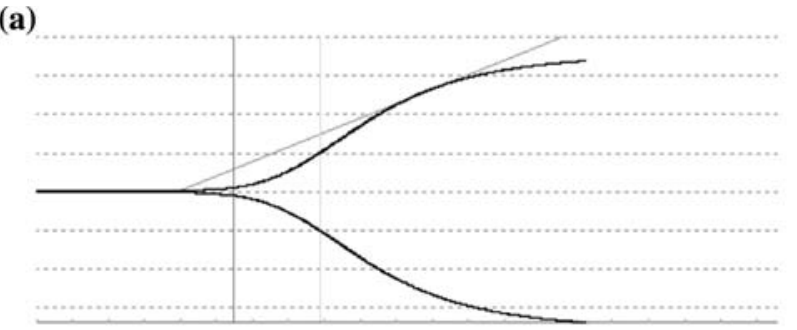

(b)

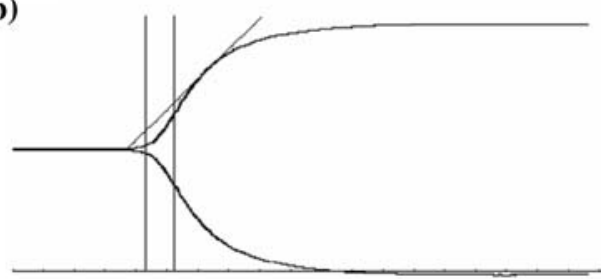

Fig. 2 Representative TEG tracings. a Hypothermic extracorporeal circuit. b Isothermic extracorporeal circuit 
Table 3 Hemodynamics, core temperature and platelet count

\begin{tabular}{llll}
\hline Variable & Group & Before & After \\
\hline MAP $(\mathrm{mmHg})$ & COOL & 84 & 86 \\
& & $(74 ; 90)$ & $(80 ; 92)$ \\
& CONTR & 80 & 82 \\
& & $(73 ; 83)$ & $(78 ; 90)$ \\
CO $(1 / \mathrm{min})$ & COOL & 5.2 & 3.9 \\
& & $(4.7 ; 5.4)$ & $(3.4 ; 4.6)$ \\
& CONTR & 5.4 & 4.1 \\
$T\left({ }^{\circ} \mathrm{C}\right)$ & & $(5.3 ; 5.5)$ & $(3.4 ; 5)$ \\
& COOL & 37.2 & 37.8 \\
& & $(36.0 ; 37.8)$ & $(37.0 ; 38.1)$ \\
& CONTR & 37.9 & 37.6 \\
PLT $\left(10^{9} / 1\right)$ & & $(37.2 ; 38.0)$ & $(35.9 ; 38.0)$ \\
& COOL & 471 & $287^{\mathrm{a}}$ \\
& & $(447 ; 518)$ & $(237 ; 374)$ \\
& CONTR & 458 & $254^{\mathrm{a}}$ \\
& & $(351 ; 505)$ & $(216 ; 296)$ \\
\hline
\end{tabular}

$M A P$ mean arterial pressure, $C O$ cardiac output, $T$ core temperature, $P L T$ platelets

a $P<0.05$ versus before

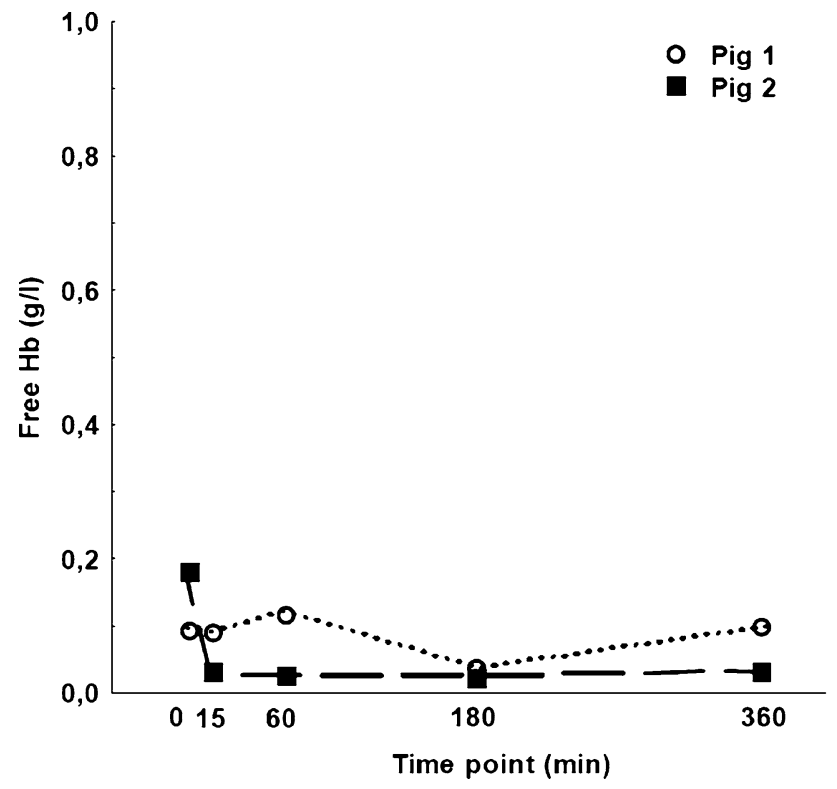

Fig. 3 Free hemoglobin in arterial plasma

randomly selected animals treated with "hypothermic circuit" CVVH (Fig. 3).

\section{Discussion}

The main result of our experimental study is that regional cooling of blood in the CRRT extracorporeal circuit to $20^{\circ} \mathrm{C}$ for $6 \mathrm{~h}$ makes it possible to perform the procedure without the need to use any anticoagulant with no blood clotting in the extracorporeal circuit.
Admittedly, our idea to use regional blood cooling in a RRT method is not completely new. This method has been employed in an experimental setting whereby pigs underwent 3-h intermittent dialysis with blood in the circuit also cooled to $20^{\circ} \mathrm{C}$ and rewarmed to $37^{\circ} \mathrm{C}$, using a microwave oven, before returning it to the animals' body [9]. However, that study was focused rather on assessing changes in biocompatibility (C5a) of intermittent dialysis modified in this manner while not seeking to monitor in detail changes in blood clotting and dynamics of thrombus formation within the circuit. Despite this, consistent with our results, the authors noted that the use of cooling allowed them to perform this relatively short procedure without in-circuit blood clotting. However, the device we have developed has a number of different features. First, it was a device intended for CRRT, i.e., a method operating at lower blood flow rates potentially associated with enhanced coagulation activation. Second, the technique of blood rewarming on return was quite different in our case (not a microwave oven but a heat exchanger, which we consider biologically safer). Moreover, using a computer, its efficacy was controlled by feedback ruling out inadequate warming of returning blood.

The explanation why extracorporeal circuit cooling helps maintain its patency with no blood clotting can be found in data regarding thrombin generation and its incircuit dynamics. These data revealed that blood cooling led primarily to inhibition of the initiation phase of thrombin generation consequently compromising the clotting process. The result was both a delay in the initiation of thrombus formation and a decrease in the rate of thrombus growth. Still, the strength of a thrombus formed in this way did not differ from that of a normal thrombus, which is a finding consistent with that reported from other studies [10]. Moreover, our results are in accordance with data published by Martini, who studied the effect of body hypothermia on coagulation in the pig [11, 12]. The role of platelet count and platelet dysfunction should also be taken into consideration when interpreting our findings. We observed a decrease in platelet count in both groups during the procedure. However, the rate of decrease did not differ between the groups and it should be noted that platelet count remained well within the physiological range, so this mechanism should not have affected the process of thrombus formation and altered its strength. Although platelet functions were not assessed in our experimental study, available data suggest that hypothermia may have a major impact on platelet functions, which, in turn, may have contributed to the anticoagulation effect of cooling seen in our study $[6,13]$.

The key issue that should be further addressed is whether multiple repetition of the process of cooling/ rewarming could have undesirable effects. It should be noted, however, that our study was not primarily designed to evaluate potential side effects, but, in the first place, to demonstrate the system functionality. To analyze the 
safety of the system, which is undoubtedly essential, would require a comprehensive approach at the levels of both cellular and non-cellular blood components (proteins, enzymes, immune system, etc.) Nevertheless, we can exclude clinically relevant hemolysis as serial free plasma hemoglobin did not change during the study. One might argue that data on free hemoglobin were obtained from two animals only. However, given their consistency and stability we do not think that getting more data from additional animals would change the picture substantially. In addition, none of the animals showed an increase in bilirubin and lactate dehydrogenase or a decrease in erythrocyte count (data not shown), further making the possibility of hemolysis unlikely.

Data regarding changes in the properties of blood initially cooled and subsequently rewarmed in terms of its ability to clot are scarce. It is known that blood rewarming results in reversal of coagulation defect [14] and rewarming a patient with hypothermic coagulopathy is a routine therapeutic intervention [15]. Likewise defect in platelets aggregation reversed after protracted hypothermia in a canine model [16]. However, none of these situation closely mimics the course of our experiment whereby cooling/ rewarming occurred after each passage of blood through the device and kept on repeating for a number of hours.

Our study has several limitations. Although testing our device in CVVH, it was not used for more than $6 \mathrm{~h}$. Still, upon completion of procedures involving blood cooling and after flushing the extracorporeal circuit with saline, we noted only minimal signs of clotting; the median of sings of clotting was 2 (using a 1-5 scale where 5 indicated complete clotting and 1 an absolutely clean system without any thrombi at all). As a result, a procedure handled in this manner could probably last for many more hours. Although similarities in the human and the porcine coagulation and fibrinolytic systems have been reported $[17,18]$, this fact should be taken into consideration in this context. In addition, our study was performed in healthy animals, but many critically ill patients scheduled for CRRT show a more or less pronounced coagulation abnormality. Therefore, given the current state of knowledge, we are unable to extrapolate the function of our device to those conditions. Similarly, the selection of temperature in the extracorporeal circuit was more or less empirical. The intention was to decrease the temperature to a level low enough, in the experience of other authors, to prevent blood from clotting [9, 13]. Judging from our results, decreasing temperature to $20^{\circ} \mathrm{C}$ is enough to obtain the desired effect; however, we are unable to say whether identical efficacy could have been obtained with a higher temperature (which would presumably also entail lower technical requirements on the device).

In summary, the method of selective in-circuit blood cooling seems to be a feasible alternative strategy for anticoagulation during CRRT, at least within this experimental setting. The crucial step for making it clinically applicable is to demonstrate its safety. Should this happen, then incorporation of the device into CRRT systems should become a technical challenge.

Acknowledgment The authors thank L. Trefil, L. Vit, and Ludmila Reifova for their skillful technical assistance. This study was supported by research grant MSM 0021620819 (Replacement of and support to some vital organs).

Open Access This article is distributed under the terms of the Creative Commons Attribution Noncommercial License which permits any noncommercial use, distribution, and reproduction in any medium, provided the original author(s) and source are credited.

\section{References}

1. Joannidis M, Oudemans-van Straaten HM (2007) Clinical review: patency of the circuit in continuous renal replacement therapy. Crit Care 11:218-227

2. Oudemans-van Straaten HM, Wester JP, de Pont AC, Schetz MR (2006) Anticoagulation strategies in continuous renal replacement therapy: can the choice be evidence based? Intensive Care Med 32:188-202

3. Tan HK, Baldwin I, Bellomo R (2000) Continuous veno-venous hemofiltration without anticoagulation in high-risk patients. Intensive Care Med 26:1652-1657

4. Simpson S, Rasmussen AT (1916) The effect of temperature on blood coagulation time. Q J Exp Physiol 10:159-168
5. Rohrer MJ, Natale AM (1992) Effect of hypothermia on the coagulation cascade. Crit Care Med 20:1402-1405

6. Wolberg AS, Meng ZH, Monroe DM, Hoffman M (2004) A systematic evaluation of the effect of temperature on coagulation enzyme activity and platelet function. J Trauma 56:12211228

7. Watts DD, Trask A, Soeken K, Perdue P, Dols S, Kaufmann C (1998) Hypothermic coagulopathy in trauma: effect of varying levels of hypothermia on enzyme speed, platelet function, and fibrinolytic activity. J Trauma 44:846-854

8. Staab DB, Sorensen VJ, Fath JJ, Raman SB, Horst HM, Obeid FN (1994) Coagulation defects resulting from ambient temperature-induced hypothermia. J Trauma 36:634-638
9. Otte KE, Jensen PB, Svendsen P, Gram J, Starklint H, Jørgensen KA (1997) Heparin-free hypothermal hemodialysis at $20^{\circ} \mathrm{C}$ improves biocompatibility. Blood Purif 15:200-207

10. Kettner SC, Sitzwohl C, Zimpfer M, Kozek SA, Holzer A, Spiss CK, Illievich UM (2003) The effect of graded hypothermia $\left(36^{\circ} \mathrm{C}-32^{\circ} \mathrm{C}\right)$ on hemostasis in anesthetized patients without surgical trauma. Anesth Analg 96:1772-1776

11. Martini WZ (2007) The effects of hypothermia on fibrinogen metabolism and coagulation function in swine. Metabolism 56:214-221 
12. Martini WZ, Pusateri AE, Uscilowicz JM, Delgado AV, Holcomb JB (2005) Independent contributions of hypothermia and acidosis to coagulopathy in swine. J Trauma 58:1002-1009

13. Kermode JC, Zheng Q, Milner EP (1999) Marked temperature dependence of the platelet calcium signal induced by human von Willebrand factor. Blood 94:199-207
14. Valeri CR, Feingold H, Cassidy G, Ragno G, Khuri S, Altschule MD (1987) Hypothermia-induced reversible platelet dysfunction. Ann Surg 205:175-181

15. DeLoughery TG (2004) Coagulation defects in trauma patients: etiology, recognition, and therapy. Crit Care Clin 20:13-24

16. Ao H, Moon JK, Tashiro M, Terasaki H (2001) Delayed platelet dysfunction in prolonged induced canine hypothermia. Resuscitation 51:83-90
17. Velik-Salchner C, Schnürer C, Fries D, Müssigang PR, Moser PL, Streif W, Kolbitsch C, Lorenz IH (2006) Normal values for thrombelastography (ROTEM) and selected coagulation parameters in porcine blood. Thromb Res 117:597-602

18. Olsen AK (1999) The pig as a model in blood and coagulation and fibrinolysis research. Scand J Lab Anim Sci 26:214-224 